\title{
A strongly nonlinear Alfvénic pulse in a transversely inhomogeneous medium
}

\author{
D. Tsiklauri, V. M. Nakariakov, and T. D. Arber
}

Physics Department, University of Warwick, Coventry, CV4 7AL, England, UK

Received 21 May 2002 / Accepted 30 August 2002

\begin{abstract}
We investigate the interaction of a plane, linearly polarized, Alfvénic pulse with a one-dimensional, perpendicular to the magnetic field, plasma density inhomogeneity in the strongly nonlinear regime. Our numerical study of the full MHD equations shows that: (i) Plasma density inhomogeneity substantially enhances (by about a factor of 2) the generation of longitudinal compressive waves. (ii) Attained maximal values of the generated transverse compressive perturbations are insensitive to the strength of the plasma density inhomogeneity, plasma $\beta$ and the initial amplitude of the Alfvén wave. Typically, they reach about $40 \%$ of the initial Alfvén wave amplitude. (iii) Attained maximal values of the generated relative density perturbations are within $20-40 \%$ for $0.5 \leq \beta \leq 2.0$. They depend upon plasma $\beta$ strongly; and scale almost linearly with the initial Alfvén wave amplitude.
\end{abstract}

Key words. magnetohydrodynamics (MHD) - waves - Sun: activity - Sun: solar wind

\section{Introduction}

The Alfvén waves are usual candidates for energy transport from the lower layers of the solar atmosphere to the corona, (e.g. Goossens 1994; Roberts 2000). However, efficient deposition of the momentum and energy require interaction of linearly incompressible Alfvén waves with compressible magnetoacoustic waves, (e.g. Ofman \& Davila 1997, 1998; Ofman et al. 2000). Also, the compressible waves, in contrast to the Alfvén waves, can transport energy and momentum across the magnetic field, spreading out the heated region. In addition, observational detection of Alfvén waves in open structures of the corona can be based upon measurement of the compressible fluctuations, (e.g. Ofman et al. 1997, 1998, 2000). These are generated in the lower corona by the Alfvén waves through linear or nonlinear mechanisms, (e.g. Nakariakov et al. 2000). This method can be complimentary to the observation of coronal Alfvén waves through non-thermal broadening of emission lines, (e.g. Banerjee et al. 1998).

In the inner heliospheric solar wind, Alfvén waves are observed in situ and represent the main component in MHD turbulence (Tu \& Marsch 1995; Tsurutani \& Ho 1999). This suggests another interesting problem: why the incompressible turbulence dominates in the solar wind, and why compressible fluctuations are not observed, despite the theoretical possibility for these two kinds of MHD fluctuations to be coupled because

Send offprint requests to: D. Tsiklauri,

e-mail: tsikd@astro.warwick.ac.uk of the medium inhomogeneity and nonlinearity. Thus, the study of coupling of compressible and incompressible fluctuations is important to the physics of the solar corona and the solar wind. There are several possible mechanisms for the coupling.

The decay instability of Alfvén waves is one of the possible examples of interaction between the MHD wave modes. This mechanism involves resonant three-wave interaction of Alfvén and magnetoacoustic waves (e.g. Sagdeev \& Galeev 1969 and references therein). The efficiency of this interaction is governed by the amplitudes of the interacting waves. However, this mechanism works only for quasi-periodic (perhaps widespectrum, Malara et al. 2000) waves, and is not efficient for the wave pulses that could be generated by some transient events on the Sun such as solar flares and coronal mass ejections, (e.g. Roussev et al. 2001).

In contrast, the efficiency of non-resonant mechanisms of the compressible fluctuation excitation by the Alfvén waves does not depend on the coherentness of Alfvén perturbation and consequently work even for single wave, wide-spectrum, pulses.

Nonlinear excitation of magnetoacoustic perturbations by nonlinear elliptically polarized Alfvén waves via the longitudinal gradients of the total pressure perturbations is one of the possible examples of the non-resonant MHD wave interaction. In this mechanism the generation of compressible perturbations results in the self-interaction and subsequent steepening of the Alfvén wave front, which is described by the Cohen-Kulsrud equation, (e.g. Cohen \& Kulsrud 1974; Verwichte et al. 1999; 
Nakariakov et al. 2000). In the following, we refer to this mechanism as "longitudinal", to distinguish it from the "transverse mechanism", which generates compressible perturbations via the transverse gradients of the total pressure perturbations in nonlinear Alfvén waves. More detailed discussion of these two mechanisms is presented in Nakariakov et al. (1997, 1998), Botha et al. (2000), Tsiklauri et al. (2001).

The transverse mechanism is dramatically modified in the case when MHD waves interact with transverse stationary inhomogeneity of the plasma. If the Alfvén speed is inhomogeneous across the magnetic field, initially plane, linearly polarized Alfvén waves become oblique and sharp gradients in the direction across the field are secularly generated. This constitutes a basis of the well known Alfvén wave phase mixing phenomenon which has been extensively investigated in connection with the solar coronal heating with MHD waves (Heyvaerts \& Priest 1983). Various aspects of this phenomenon have been intensively studied using full MHD numerical simulations (Malara et al. 1996; Ofman \& Davila 1995, 1997; Poedts et al. 1997; Grappin et al. 2000; De Moortel et al. 2000). As demonstrated by Nakariakov et al. (1997, 1998); Botha et al. (2000); Tsiklauri et al. (2001), phase mixing of Alfvén waves in the compressible plasma, in the weakly nonlinear regime leads to the generation of fast magnetoacoustic waves, and various regimes of this process, relevant to solar coronal and heliospheric applications have been studied. In particular, it has been found that the inhomogeneity of the plasma across the magnetic field, associated with various types of structuring (e.g. plumes in the coronal holes, boundaries between the slow and the fast solar winds, flow tubes, etc.), plays the crucial role in the interaction of compressible and incompressible weaklynonlinear MHD modes.

Problems connected with the interpretation of MHD fluctuations observed in the solar wind and the propagation of intensive MHD wave pulses in the solar corona (e.g., flare and CME-associated waves) require detailed study of the interaction of the MHD pulses with plasma inhomogeneities in the strongly nonlinear regime. Our interest to this regime is motivated by the necessity to account for effects of higher order nonlinearities and the back-reaction of nonlinearly generated compressible perturbations on the source Alfvén pulse. Also, as the efficiency of the "transverse" mechanism is connected to the longitudinal wave number (together with the transverse wave number and the amplitude), and as the "longitudinal" mechanism increases, through wave steepening, the longitudinal wave numbers, we expect that the simultaneous action of these two mechanisms can enhance the efficiency of the transverse mechanism. In this work, we study, by direct numerical simulations, the generation of compressible fluctuations by a strongly nonlinear Alfvénic pulse interacting with a transverse plasma inhomogeneity.

The paper is organized as follows: in Sect. 2 we describe our model and the numerical method applied, in Sect. 3 the results of the simulations are discussed separately in the high and low $\beta$ cases (Sects. 3.1 and 3.2 respectively), Sect. 3.3 deals with the investigation of parametric space, and finally, the conclusions are presented in Sect. 4.

\section{The model}

The model studied here is similar to one discussed in Tsiklauri et al. (2001): we use the equations of ideal MHD

$$
\begin{aligned}
& \rho \frac{\partial \boldsymbol{V}}{\partial t}+\rho(\boldsymbol{V} \cdot \nabla) \boldsymbol{V}=-\nabla p-\frac{1}{4 \pi} \boldsymbol{B} \times \operatorname{curl} \boldsymbol{B}, \\
& \frac{\partial \boldsymbol{B}}{\partial t}=\operatorname{curl}(\boldsymbol{V} \times \boldsymbol{B}), \\
& \frac{\partial p}{\partial t}+\boldsymbol{V} \cdot \nabla p+\gamma p \nabla \cdot \boldsymbol{V}=0, \\
& \frac{\partial \rho}{\partial t}+\operatorname{div}(\rho \boldsymbol{V})=0,
\end{aligned}
$$

where $\boldsymbol{B}$ is the magnetic field, $\boldsymbol{V}$ is plasma velocity, $\rho$ is plasma mass density, and $p$ is plasma thermal pressure for which the adiabatic variation law is assumed.

We solve Eqs. (1)-(4) in Cartesian coordinates $(x, y, z)$ and under the assumption that there is no variation of the physical values in the $y$-direction, i.e. $(\partial / \partial y=0,2.5 \mathrm{D}$ approximation) with the use of Lare2d (Arber et al. 2001). Lare2d is a numerical code which operates by taking a Lagrangian predictorcorrector time step and after each Lagrangian step all variables are conservatively re-mapped back onto the original Eulerian grid using van Leer gradient limiters. This code was also used to produce the results in Botha et al. (2000), Tsiklauri et al. (2001). As in Botha et al. (2000), Tsiklauri et al. (2001), the equilibrium state is taken to be a uniform magnetic field $B_{0}$ in the $z$-direction and an inhomogeneous plasma of density $\rho_{0}(x)$,

$\rho_{0}(x)=\rho_{*}(3-2 \tanh (\lambda x))$.

Here, $\lambda$ is a free parameter which controls the steepness of the density profile gradient. The latter is localized around $x=0$. The temperature profile $T_{0}(x)$ is set up to allow for the total pressure to be constant everywhere. In our normalization, which is the same as that of Botha et al. (2000); Tsiklauri et al. (2001), $\boldsymbol{B}=B_{0} \overline{\boldsymbol{B}}, \boldsymbol{r}=a_{*} \overline{\boldsymbol{r}}, C_{\mathrm{A}}(x)=B_{0} / \sqrt{4 \pi \rho_{0}(x)}=$ $\left[B_{0} / \sqrt{4 \pi \rho_{*}}\right] / \sqrt{3-2 \tanh (\lambda x)}=C_{\mathrm{A}}^{*} / \sqrt{3-2 \tanh (\lambda x)}=$ $C_{\mathrm{A}}^{*} \bar{C}_{\mathrm{A}}(x), t=\left(a_{*} / C_{\mathrm{A}}^{*}\right) \bar{t}, \boldsymbol{V}=C_{\mathrm{A}}^{*} \overline{\boldsymbol{V}}$, the dimensionless local Alfvén speed is $\bar{C}_{\mathrm{A}}(x)=1 / \sqrt{3-2 \tanh (\lambda x)} . a_{*}$ and $\rho_{*}$ are the units of length and density respectively. In what follows we omit bars on all dimensionless physical quantities.

\section{Numerical results}

We set up the code in such a way that initially longitudinal $\left(V_{z}\right)$ and transverse $\left(V_{x}, B_{x}, B_{z}\right)$ perturbations and the density perturbation, $\rho$, (perturbed by both longitudinal and transverse modes) are absent and the initial amplitude of the Alfvén pulse is strongly non-linear, i.e. typically $A=0.5$. At $t=0$, the Alfvén perturbation is a plane (with respect to $x$-coordinate) pulse, which has a Gaussian structure in the $z$-coordinate,

$B_{y}(z)=A \exp \left(-\frac{z^{2}}{\delta}\right), V_{y}(x, z)=-C_{\mathrm{A}}(x) B_{y}(z)$.

Here, $\delta$ is a free parameter which controls the width of the initial pulse. In all our numerical runs $\delta=0.1$.

As it is discussed in Introduction (cf. Nakariakov et al. (1997) for details) in the considered geometry, Alfvén waves 
represented by $V_{y}$ and $B_{y}$, are incompressible up to the $c u$ bic nonlinearity. The longitudinal (represented by $V_{z}$ ) and the transverse (represented by $V_{x}, B_{x}, B_{z}$ ) compressible perturbations (of course, both perturbing $\rho$ ) are generated nonlinearly by the longitudinal and transverse gradients of the total pressure. The efficiency of the generation depends upon the amplitude of the Alfvén waves, in other words, the incompressible and compressible perturbations are linearly decoupled. The latter guarantees that with the choice of our initial conditions the compressible perturbations are indeed initially absent from the system, which a priori is not clear if the linear coupling is present (cf. Tsiklauri \& Nakariakov 2002). In addition, the transverse compressible perturbations are generated by plane Alfvén waves only in the presence of the transverse profile of the local Alfvén speed.

The simulation box size is set by the limits $-15.0<x<$ 15.0 and $-15.0<z<15.0$. The pulse starts to move from point $z=-12.5$ towards the positive $z$ 's. Both the scale of density inhomogeneity and width of the Alfvénic pulse are much smaller than the size of the calculation domain.

We have performed calculations on various resolutions in an attempt to achieve convergence of the results. The graphical results presented here are for the spatial resolution $2500 \times$ 2500 , which refers to number of grid points in $z$ and $x$ directions respectively. We have used a non-uniform grid in our simulations, namely, in $x$ direction $75 \%$ of the grid points where concentrated between $-5.0 \leq x \leq 5.0$ where the spatial inhomogeneity has strongest gradients. We have also performed calculation on the spatial resolution $3500 \times 3500$ and found that the maximal generation levels (maximum of an absolute value over the whole simulation domain) for all physical quantities as a function of time are the same as in the case of $2500 \times 2500$ resolution. Thus, the results presented here are, indeed, converged.

Our main numerical runs are split in two parts, treating separate cases when plasma $\beta$, which is the ratio of speed of sound to the Alfvén velocity squared, is less and greater than unity. This split is motivated by weakly nonlinear results, showing that the particular scenario of the interaction of MHD waves is determined by the ratio $\left(C_{\mathrm{s}} / C_{\mathrm{A}}\right)$ being less or greater then unity, (e.g. Cohen \& Kulsrud 1974).

\subsection{Case when $\beta>1$}

In this subsection we present solution of the Eqs. (1)-(4) with the above described equilibrium and the initial conditions for the case when plasma- $\beta$ is 2.0 . Here, $\lambda$-parameter was fixed at 0.75 .

Figures 1-3 show snapshots of the initially absent transverse $\left(V_{x}\right)$ and longitudinal $\left(V_{z}\right)$ components of the velocity (representing the "transverse" and "longitudinal" compressible waves, respectively) and density perturbation, at time $t=15$.

The transverse compressible perturbations $\left(V_{x}\right)$ are generated by the transverse gradients of the total pressure perturbations. In turn, these perturbations are generated by the Alfvén wave phase mixing, and are located near $x=0$ where the density gradients are the largest. Then they propagate across the field (see Fig. 1). The longitudinal compressive

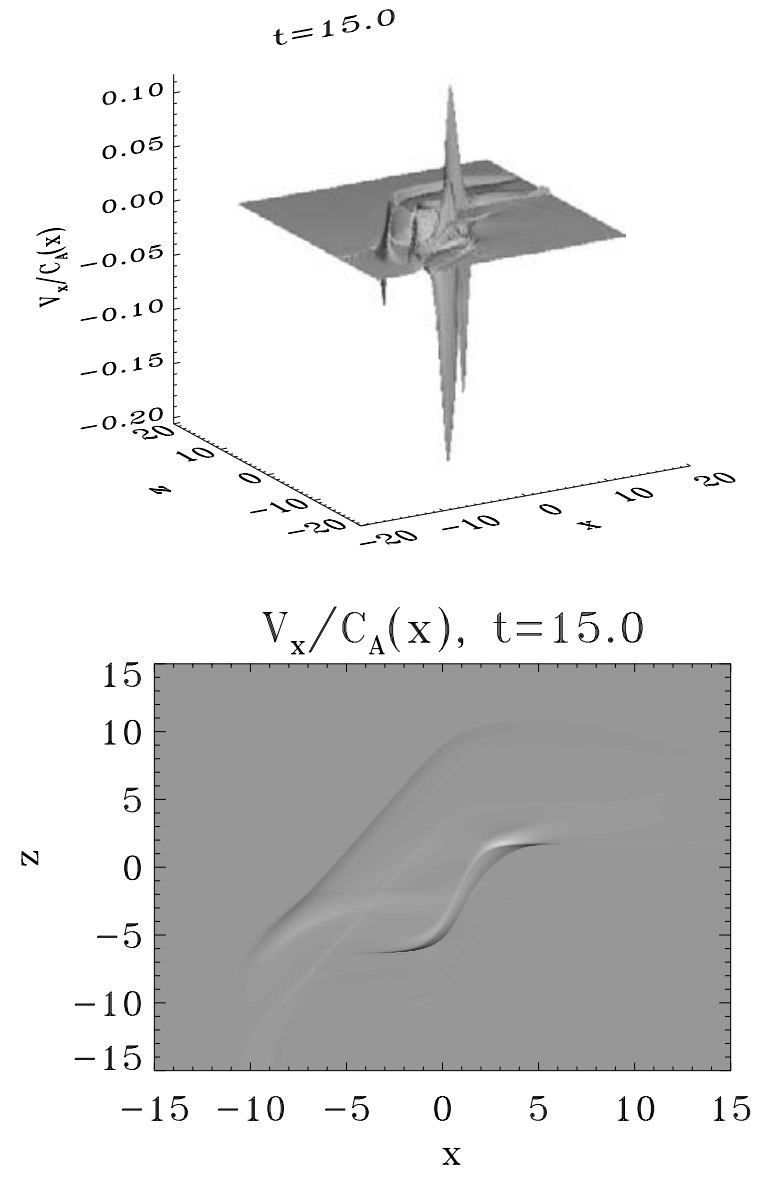

Fig. 1. Top panel: snapshot of $V_{x} / C_{\mathrm{A}}(x)$ at $t=15.0$. Bottom panel: contour-plot of $V_{x} / C_{\mathrm{A}}(x)$ at the same instance. Here, initial amplitude, $A=0.5$, density inhomogeneity steepness, $\lambda=0.75$, plasma $\beta=2$.

perturbations, $V_{z}$ are generated even in the absence of the plasma density inhomogeneity (Fig. 2). In the contour plot (Fig. 2, bottom panel) it can be seen that there are two wave fronts: one moving at a local Alfvén speed, and another (compressive one) that moves faster than the local Alfvén speed because $\beta=2$. Figure 3 shows the relative density perturbation associated with the compressible waves that have been generated.

In Fig. 4, the top panel presents spatial variation (across the $x$-coordinate) and the dynamics of the transverse and longitudinal compressible waves, and relative density perturbations produced by them. In what follows max and min refer to the maximum and minimum over the whole simulation box (i.e. space) of a dimensionless physical quantity at a given time instance, respectively. These are fairly good and simple (scalar) quantities describing the generation and/or decay of a $2.5 \mathrm{D}$ physical quantity, dynamics of which is otherwise not so straightforward to comprehend. There are five interesting observations: (i) the decay of the $\max \left(\left|V_{y}\right| / C_{\mathrm{A}}(x)\right.$ ) (on expense of which the transverse $\left(V_{x}\right)$ and longitudinal $\left(V_{z}\right)$ compressive waves and associated density perturbation are generated) occurs in the middle - where the plasma inhomogeneity is the strongest. This demonstrates the importance of the inhomogeneity. (ii) the right wing $(x>0)$ decays faster than the left $(x<0)$ one. For the value of plasma $\beta$ used, it is expected that shock 

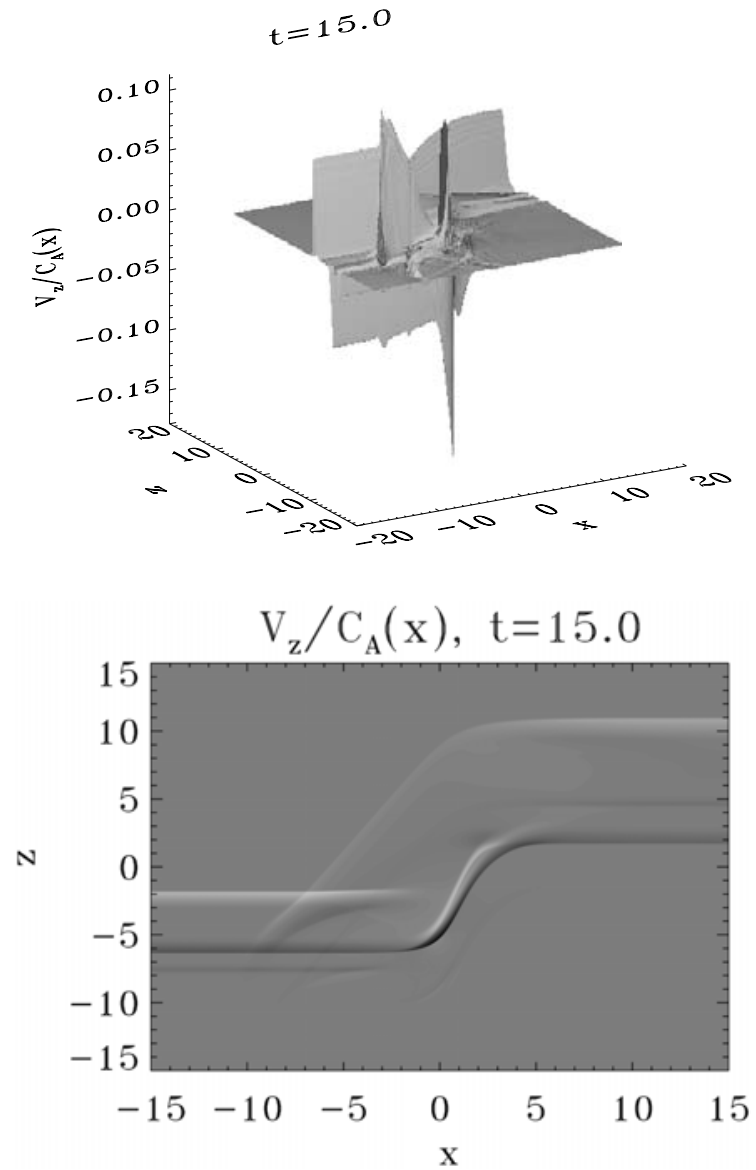

Fig. 2. Top panel: snapshot of $V_{z} / C_{\mathrm{A}}(x)$ at $t=15.0$. Bottom panel: contour-plot of $V_{z} / C_{\mathrm{A}}(x)$ at the same instance. Here, the parameters are the same is in Fig. 1.

dissipation would be greater where the local Alfvén velocity is greater (in this case the right wing). Note that when referring to shock dissipation we mean artificial dissipation that guarantees proper shock capturing, while at all times we remain in the framework of an ideal MHD (no bulk dissipation included). Thus, the shock viscosity ensures that we recover the weak solution to ideal MHD. (iii) the relative density perturbation and longitudinal compressive wave $\max \left(\left|V_{z}\right| / C_{\mathrm{A}}(x)\right)$ are generated in the homogeneous parts of the domain $(x \leq-5, x \geq 5)$ too. However, in the middle of the domain, where the inhomogeneity is strong we see that inhomogeneity of plasma density enhances the generation of these quantities by about a factor of 2. (iv) transverse compressive wave $\max \left(\left|V_{x}\right| / C_{\mathrm{A}}(x)\right)$, which is not generated in the absence of density inhomogeneity, is generated in the middle of the domain. (v) $\max \left(\left|V_{y}\right| / C_{\mathrm{A}}(x)\right)$, on expense of which the transverse $\left(\max \left(\left|V_{x}\right| / C_{\mathrm{A}}(x)\right)\right)$ and longitudinal $\left(\max \left(\left|V_{z}\right| / C_{\mathrm{A}}(x)\right)\right)$ compressive waves are generated, has two dips in those places where the other physical quantities have two bumps, which clearly demonstrates the correct energy balance as well as the importance of the inhomogeneity (where actually the bumps occur).

In Fig. 4, the bottom panel presents temporal variation of transverse and longitudinal compressible wave amplitudes. In the presence of the inhomogeneity $(\lambda=0.75)$, the Alfvén perturbation $\left(\min \left(\left|V_{y}(x, z, t)\right| / C_{\mathrm{A}}(x)\right)\right)$ decays much faster than in
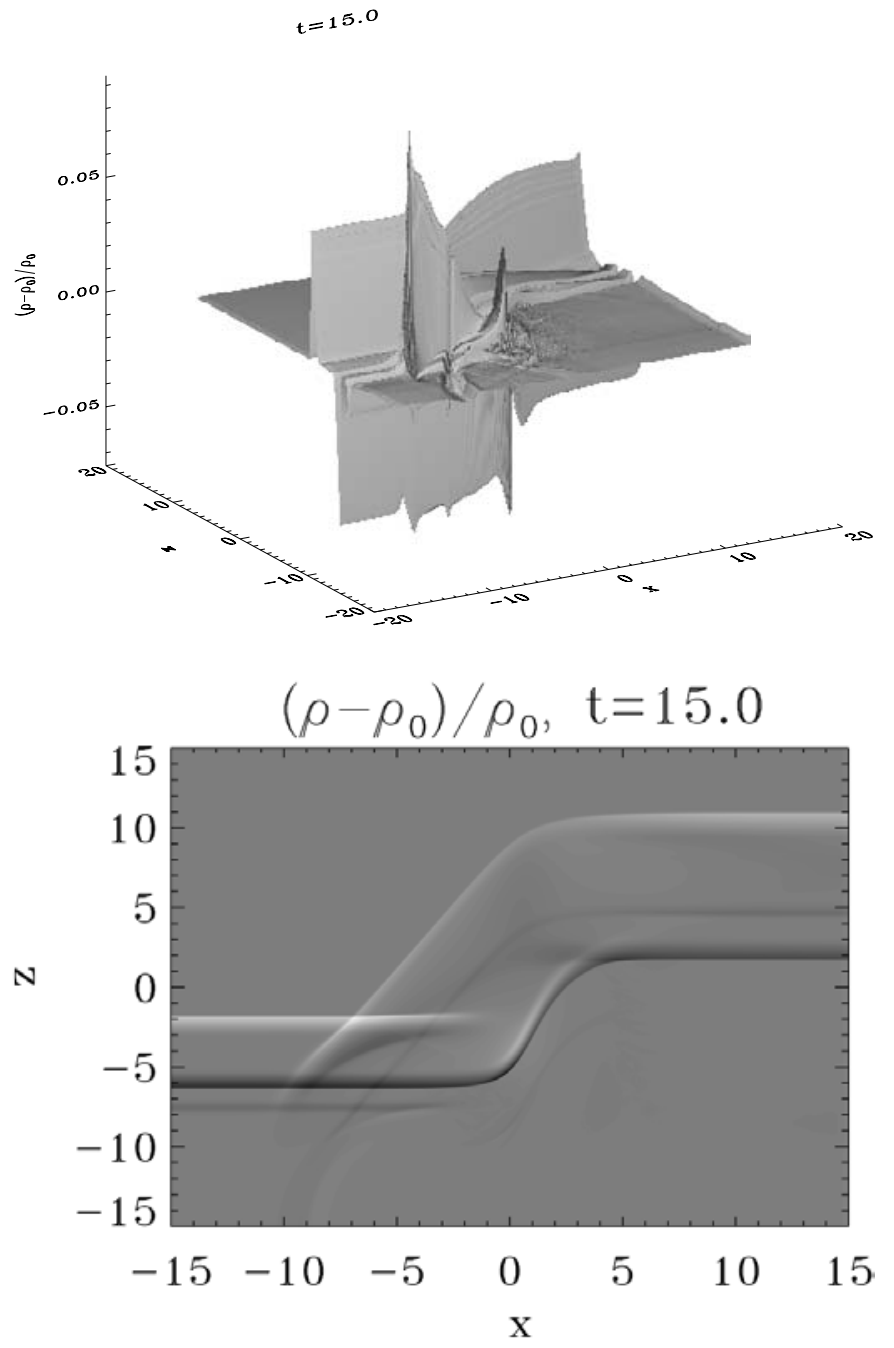

Fig. 3. Top panel: snapshot of $\left(\rho-\rho_{0}\right) / \rho_{0}$ at $t=15.0$. Bottom panel: contour-plot of $\left(\rho-\rho_{0}\right) / \rho_{0}$ at the same instance. Here, the parameters are the same is in Fig. 1.

the homogeneous case $(\lambda=0)$. In the inhomogeneous case, the energy initially stored in the Alfvén wave in addition to shock dissipation goes into the generation of the compressive waves. The maximum amplitudes of longitudinal $\max \left(\left|V_{z}(x, z, t)\right|\right)$ and transverse $\max \left(\left|V_{x}(x, z, t)\right|\right)$ compressible waves attain a substantial fraction of the initial Alfvén wave amplitude. In the inhomogeneous case $(\lambda=0.75)$, the longitudinal wave attains about twice the maximal value than in the homogeneous plasma case. Obviously, when $\lambda=0$ there is no generation of the transverse compressive wave and $\max \left(\left|V_{x}(x, z, t)\right|\right)$ is identically zero for all times.

\subsection{Case when $\beta<1$}

In this subsection we present solution of the Eqs. (1)-(4) with the above described equilibrium and the initial conditions for the case when plasma- $\beta$ is 0.5 . As in the previous subsection, $\lambda$-parameter was fixed at 0.75 .

Again, the initially absent transverse $\left(V_{x}\right)$ and longitudinal $\left(V_{z}\right)$ compressive waves and density perturbations are 

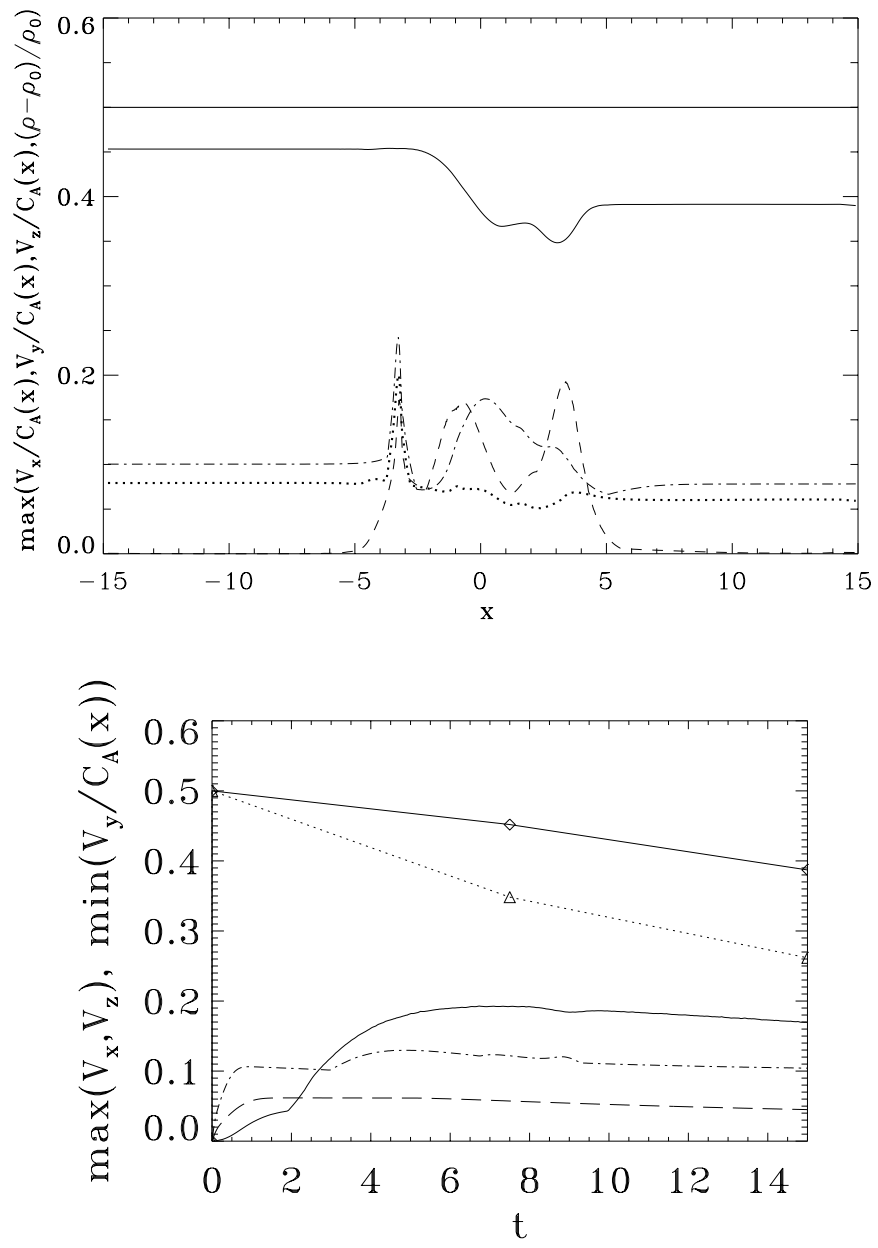

Fig. 4. Top panel: spatial variation (across $x$-coordinate) and the dynamics of non-linear generation of the transverse and longitudinal compressive waves as well as relative density perturbation in time. Thick solid line corresponds to the initial value (at $t=0$ ) of $\max \left(\left|V_{y}\right| / C_{\mathrm{A}}(x)\right)$. Thin solid curve represents the same, but for $t=7.5$. The relative density perturbation $\max \left(\left|\left(\rho-\rho_{0}\right) / \rho_{0}\right|\right)$ (dotted curve), the longitudinal compressive wave $\max \left(\left|V_{z}\right| / C_{\mathrm{A}}(x)\right)$ (dashdotted curve), and the transverse compressive wave $\max \left(\left|V_{x}\right| / C_{\mathrm{A}}(x)\right)$ (dashed curve) are given for $t=7.5$. Bottom panel: Evolution of $\max \left(\left|V_{x}(x, z, t)\right|\right), \min \left(\left|V_{y}(x, z, t)\right| / C_{\mathrm{A}}(x)\right), \max \left(\left|V_{z}(x, z, t)\right|\right)$ in time. The solid curve with open rectangles presents decay of the initial Alfvén perturbation $\left(\min \left(\left|V_{y}(x, z, t)\right| / C_{\mathrm{A}}(x)\right)\right)$ due to shock dissipation in the case of absence of the plasma density inhomogeneity $(\lambda=0)$ for three time instances $(t=0,7.5,15.0)$. The dotted curve with open triangles depicts the same, but when $\lambda=0.75$. The dash-dotted curve represents $\max \left(\left|V_{z}(x, z, t)\right|\right)$, while thick solid curve corresponds to $\max \left(\left|V_{x}(x, z, t)\right|\right)$ both for the case of $\lambda=0.75$. The long dashed curve represents $\max \left(\left|V_{z}(x, z, t)\right|\right)$ for the case when $\lambda=0$. Here, the parameters are the same is in Fig. 1.

efficiently generated (Figs. 5-7) and reach a substantial fraction of the initial Alfvén wave amplitude.

In the contour plots in Figs. 6 and 7 it can be seen that there are two wave fronts: one moving at a local Alfvén speed, and another (compressive one) that moves slower than the local Alfvén speed because $\beta=0.5$ (compare with Figs. 2 and 3). Therefore, since the velocity difference between the first and
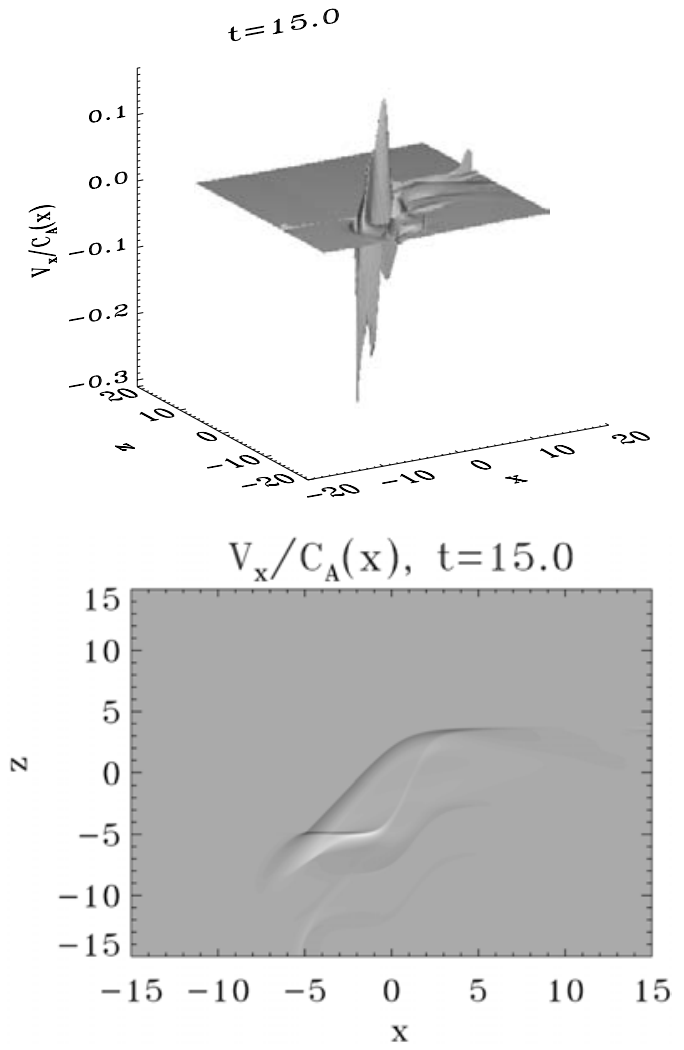

Fig. 5. Top panel: snapshot of $V_{x} / C_{\mathrm{A}}(x)$ at $t=15.0$. Bottom panel: contour-plot of $V_{x} / C_{\mathrm{A}}(x)$ at the same instance. Here, initial amplitude, $A=0.5$, density inhomogeneity steepness, $\lambda=0.75$, plasma $\beta=0.5$.
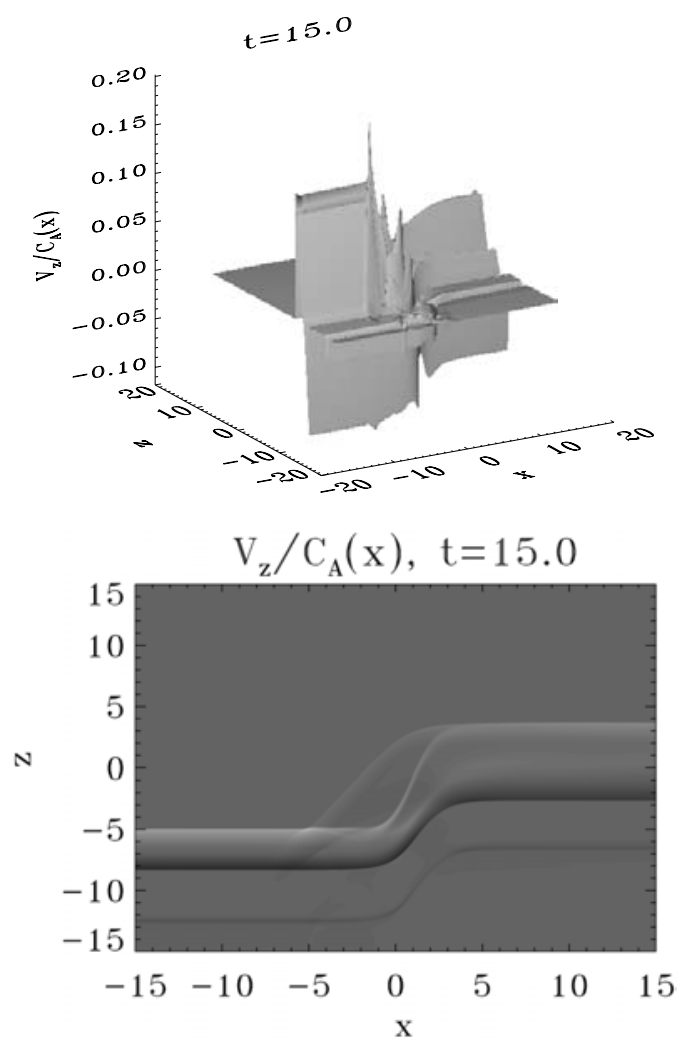

Fig. 6. Top panel: snapshot of $V_{z} / C_{\mathrm{A}}(x)$ at $t=15.0$. Bottom panel: contour-plot of $V_{z} / C_{\mathrm{A}}(x)$ at the same instance. Here, the parameters are the same is in Fig. 5. 

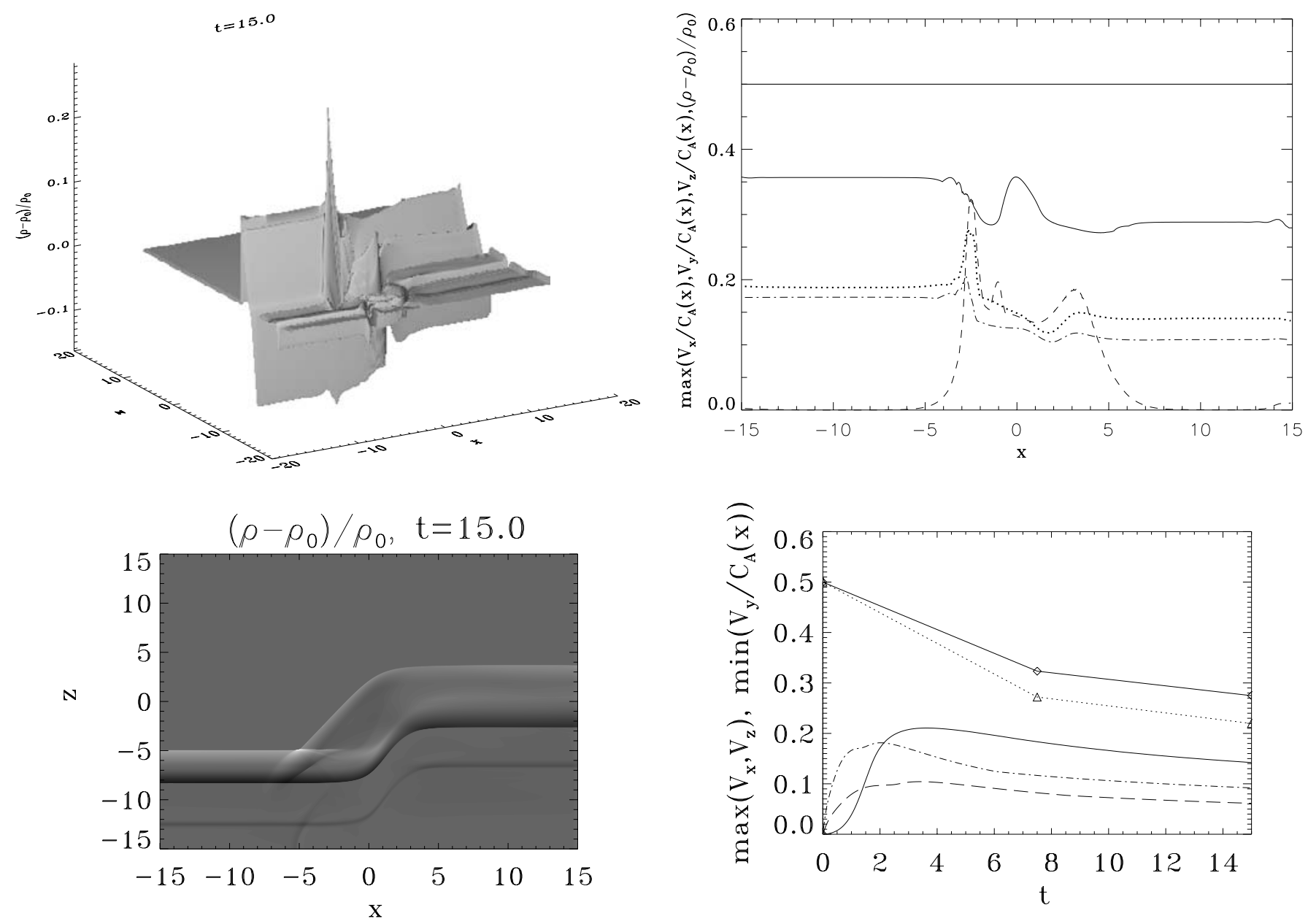

Fig. 7. Top panel: snapshot of $\left(\rho-\rho_{0}\right) / \rho_{0}$ at $t=15.0$. Bottom panel: contour plot of $\left(\rho-\rho_{0}\right) / \rho_{0}$ at the same instance. Here, the parameters are the same is in Fig. 5.

second parts of the solution is not as great as in the $\beta=2$ case, these two parts seem be blended into each other.

Figure 8 presents the evolution of transverse and longitudinal compressive wave amplitudes in time. This graph is quite similar to Fig. 4. The noteworthy difference is as follows: for the value of plasma $\beta$ used here $(\beta=0.5)$, the non-linear term in the scalar Cohen-Kulsrud equation is larger than in the former case of $\beta=2$ (Fig. 4). Therefore in Fig. 8, top panel, we observe that the shock dissipation of the Alfvén wave is greater (the thin solid line goes further down than in Fig. 4, also the top panel), and, in turn, the non-linear generation of the transverse and longitudinal compressive is further enhanced.

\subsection{Parametric study}

In this subsection we explore the parametric space of the problem. In particular, we investigate how the maximal value of the generated transverse compressive wave depends on the plasma density inhomogeneity parameter, $\lambda$, plasma $\beta$, and initial amplitude of the Alfvén wave, $A$.

In Fig. 9a we plot the dependence of the maximum of the absolute value of the transverse compressive perturbation, $\max \left(\left|V_{x}(x, z, t)\right|\right) / A$, versus $\lambda$ for $\beta=2$ (solid curve) and

Fig. 8. The same as in Fig. 4, but with $\beta=0.5$.

$\beta=0.5$ (dashed curve). There are two noteworthy features in this graph, first, the maximal value of the generated transverse compressive wave depends on the plasma density inhomogeneity parameter rather weakly (once $\lambda \geq 0.4$ ), and second, efficiency of the generation of $V_{x}$ is somewhat larger in the $\beta=0.5$ case than in the case of $\beta=2$. There are no data points between $0 \leq \lambda \leq 0.4$ because in order to accommodate the inhomogeneity in the simulation domain we would have to increase its size, which was not possible with available computational resources. Also, we made 5 runs of Lare $2 d$ code for the different values of $\beta$. The results are presented in Fig. 9b. We gather from the graph that the maximal value of the generated transverse compressive wave depends on the plasma $\beta$ rather weakly.

Yet another valuable insight can be obtained by studying the dependence of the maximal value of generated transverse compressive wave on the initial amplitude of the Alfvén wave as our problem is essentially non-linear. In Fig. 9c we plot results of numerical runs for different values of $A$, while $\lambda$ and $\beta$ where fixed at 0.5 and 1.25 respectively. We gather from Fig. 9c that quite unexpectedly the ratio $\max \left(\left|V_{x}(x, z, t)\right|\right) / A$ is insensitive to the variation of the initial amplitude of the Alfvén wave.

We also investigated the parametric space with regard to the relative density perturbation. Namely, we investigate dependence of $\max \left(\left|\left(\rho-\rho_{0}\right) / \rho_{0}\right|\right)$ as function of $\lambda, \beta$ and $A$. We gather from Fig. 9d that for $\beta=2$ the generated relative 

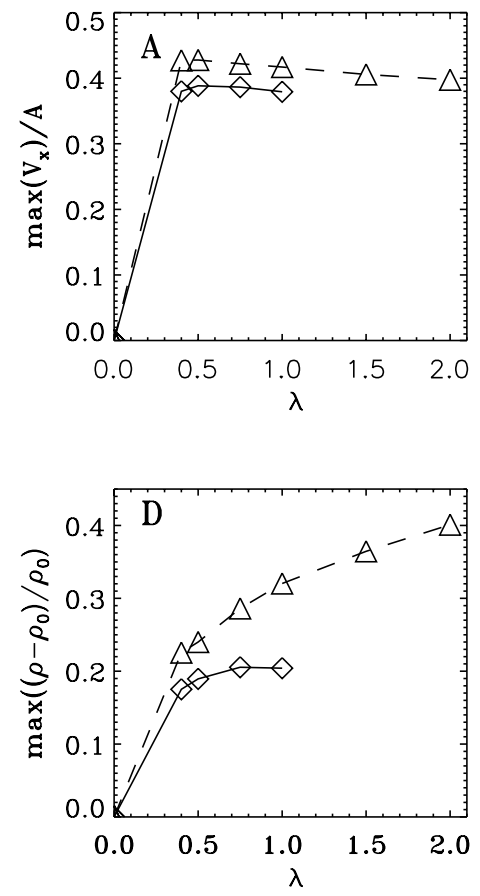
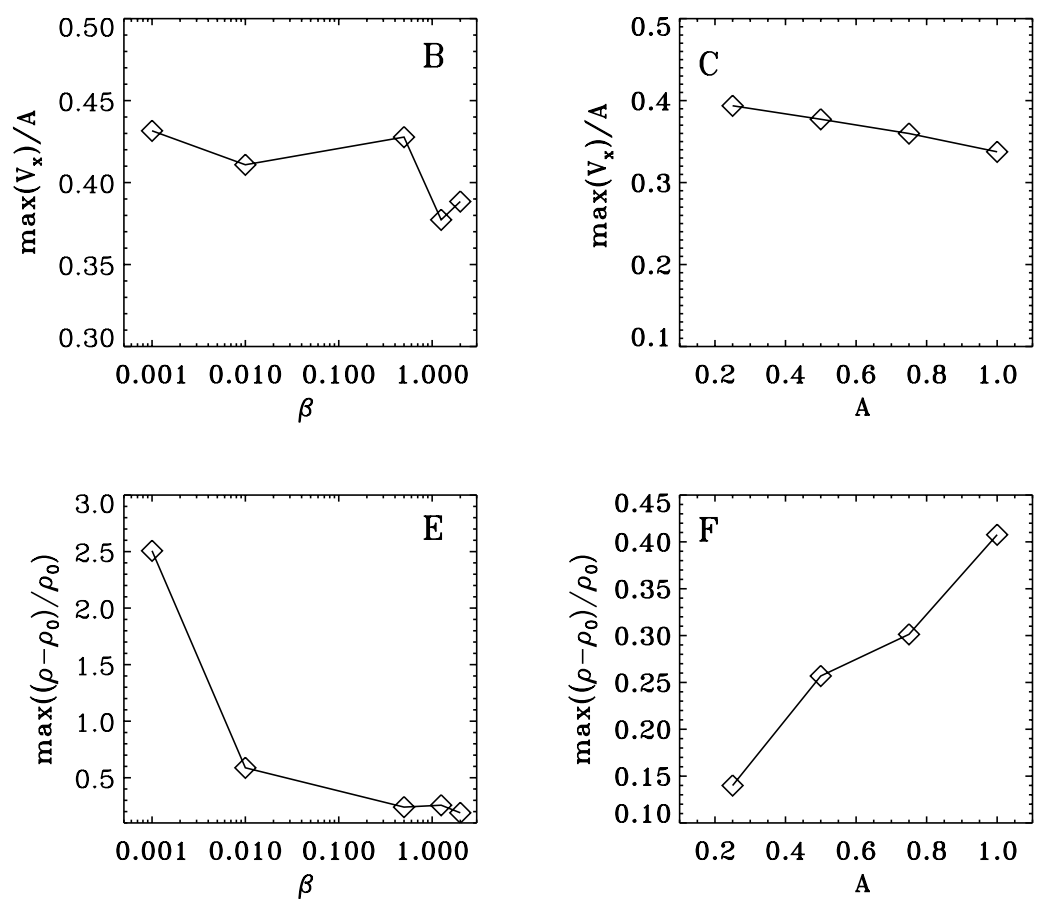

Fig. 9. a) Dependence of $\max \left(\left|V_{x}(x, z, t)\right|\right) / A$ versus $\lambda$ (density inhomogeneity steepness) for $\beta=2$ (solid curve) and $\beta=0.5$ (dashed curve). Here, initial amplitude $A=0.5$. b) The same, but versus $\beta$ for $\lambda=0.5$ and $A=0.5$. c) The same, but versus $A$ for $\lambda=0.5$ and $\beta=1.25$. d) Dependence of $\max \left(\left|\left(\rho-\rho_{0}\right) / \rho_{0}\right|\right)$ versus $\lambda$ for $\beta=2$ (solid curve) and $\beta=0.5$ (dashed curve) both for $A=0.5$. e) The same, but versus $\beta$ for $\lambda=0.5$ and $A=0.5$. f) The same, but versus $A$ for $\lambda=0.5$ and $\beta=1.25$.

density perturbation saturates at about $20 \%$, while for $\beta=0.5$ the saturation level doubles. Figure 9e illustrates the fact that the maximum generated relative density perturbation depends quite strongly on plasma $\beta$. In Fig. 9f we show the dependence of $\max \left(\left|\left(\rho-\rho_{0}\right) / \rho_{0}\right|\right)$ versus the initial amplitude $A$. We observe that since the density perturbation is generated by the non-linear effects, $\max \left(\left|\left(\rho-\rho_{0}\right) / \rho_{0}\right|\right)$ indeed grows with the increase of $A$, and the dependence is almost linear.

\section{Conclusions}

This study is an extension of the previous works to the case of strongly nonlinear amplitudes. The main results can be summarized as follows:

- Phase mixing of a strongly nonlinear Alfvén pulse is accompanied by an enhanced generation of compressible waves. This is true irrespective of plasma $\beta$ being less or greater than unity.

- Plasma density inhomogeneity (while providing, as in the weakly nonlinear case, a source region for the non-linear generation of transverse compressive waves by a plane Alfvén wave) substantially enhances (by about a factor of 2) the generation of longitudinal compressive waves.

- Attained maximal values of the generated transverse compressive perturbations are insensitive to the strength of the plasma density inhomogeneity, plasma $\beta$ and the initial amplitude of the Alfvén wave. Typically, they reach about $40 \%$ of the initial Alfvén wave amplitude.

- Attained maximal values of the generated relative density perturbations are within $20-40 \%$ for $0.5 \leq \beta \leq 2.0$.
They depend upon plasma $\beta$ strongly; and scale (increase) almost linearly with the initial Alfvén wave amplitude.

Commenting upon the maximal values attained by the transverse compressive waves in relation to the previous, weakly nonlinear, results (Botha et al. 2000; Tsiklauri et al. 2001) we would like to state the following: In the weakly nonlinear case the transverse compressive waves do not grow to a substantial fraction of the initial Alfvén wave amplitude due to the destructive wave interference effect (typically, $\max \left(\left|V_{x}(x, z, t)\right|\right) / A \sim$ $\left.10^{-3}\right)$. However, in the strongly nonlinear case, studied here, although the destructive wave interference is still in action, $\max \left(\left|V_{x}(x, z, t)\right|\right) / A$ is now significantly larger, about 0.4 (cf. Figs. $9 \mathrm{a}-\mathrm{c})$. Therefore, we conclude that the strong nonlinearity substantially enhances the attained maximal values of the transverse compressive waves.

The physical phenomenon studied here is an elementary process responsible for non-resonant coupling of compressible and incompressible MHD modes. In particular, it may play a role in MHD turbulence of space and astrophysical plasmas. The presence of a pressure-balanced inhomogeneity should significantly affect the saturated MHD turbulent state (cf. a similar claim but based upon different reasonings in Bhattacharjee et al. 1998).

Acknowledgements. D.T. acknowledges financial support from PPARC. Numerical calculations of this work were performed using the PPARC funded Compaq MHD Cluster at St Andrews and AstroSun cluster at Warwick. 


\section{References}

Arber, T. D., Longbottom, A. W., Gerrard, C. L., \& Milne, A. M. 2001, J. Comput. Phys., 171, 151

Banerjee, D., Teriaca, L., Doyle, J. G., \& Wilhelm, K. 1998, A\&A, 339,208

Bhattacharjee, A., Ng, C. S., \& Spangler, S. R. 1998, ApJ, 494, 409

Botha, G. J. J., Arber, T. D., Nakariakov, V. M., \& Keenan, F. P. 2000, A\&A, 363, 1186

Cohen, R. H., \& Kulsrud, R. M. 1974, Phys. Fluids, 17, 2215

De Moortel, I., Hood, A. W., \& Arber, T. D. 2000, A\&A, 354, 334

Goossens, M. 1994, Space Sci. Rev., 68, 51

Grappin, R., Léorat, J., \& Buttighoffer, A. 2000, A\&A, 362, 342

Heyvaerts, J., \& Priest, E. R. 1983, A\&A, 117, 220

Malara, F., Primavera, L., \& Veltri, P. 1996, ApJ, 459, 347

Malara, F., Primavera, L., \& Veltri, P. 2000, Phys. Plasmas, 7, 2866

Nakariakov, V. M., Ofman, L., \& Arber, T. D. 2000, A\&A, 353, 741

Nakariakov, V. M., Roberts, B., \& Murawski, K. 1997, Sol. Phys., 175,93

Nakariakov, V. M., Roberts, B., \& Murawski, K. 1998, A\&A, 332, 795

Ofman, L., \& Davila, J. M. 1995, J. Geophys. Res., 100, 23413

Ofman, L., \& Davila, J. M. 1997, ApJ, 476, 357

Ofman, L., \& Davila, J. M. 1998, J. Geophys. Res., 103, 23677
Ofman, L., Nakariakov, V. M., \& Sehgal, N. 2000, ApJ, 533, 1071

Ofman, L., Romoli, M., Poletto, G., Noci, G., \& Kohl, J. L. 1997, ApJ, 491, L111

Ofman, L., Romoli, M., Poletto, G., Noci, G., \& Kohl, J. L. 1998, ApJ, 507, L189

Ofman, L., Romoli, M., Poletto, G., Noci, G., \& Kohl, J. L. 2000, ApJ, 529,592

Poedts, S., Toth, G., \& Belien, A. J. C., \& Goedbloed, J. P. 1997, Sol. Phys., 172, 45

Roberts, B. 2000, Sol. Phys., 193, 139

Roussev, I., Galsgaard, K., Erdelyi, R., \& Doyle, J. G. 2001, A\&A, 370, 298

Sagdeev, R. Z., \& Galeev, A. A. 1969, Nonlinear Plasma Theory (Benjamin, New York)

Tsiklauri, D., Arber, T. D., \& Nakariakov, V. M. 2001, A\&A, 379, 1098

Tsiklauri, D., \& Nakariakov, V. M. 2002, A\&A, 393, 321

Tsurutani, B. T., \& Ho, C. M. 1999, Rev. Geophys., 37, 517

Tu, C.-Y., \& Marsch, E. 1995, Space Sci. Rev., 73, 1

Verwichte, E., Nakariakov, V. M., \& Longbottom, A. 1999, J. Plasma Phys., 62, 219 